\title{
A NOTE ON UNIQUENESS FOR ANISOTROPIC FLUIDS
}

\author{
by R. N. HILLS \\ (Received 16 August 1972)
}

1. Introduction. In 1960 Ericksen [1] introduced a simple theory of anisotropic fluids. This theory differs from the classical theory of fluids in that the deformation of the material is no longer solely described by the usual vector displacement field but requires in addition the specification of a further vector field $d_{i}$, termed the director. Moreover, corresponding to this increased kinematic flexibility new types of stress, body force and inertia are introduced. Leslie [2], adopting the conservation laws of [1], formulated constitutive equations similar to those considered by Ericksen and discussed the thermodynamical restrictions imposed by the Clausius-Duhem inequality. Here we shall consider the case in which at each point the director is constrained to remain a unit vector. Then the usual interpretation is to regard $d_{i}$ as indicating a single preferred direction in the material (see for example [3]). It is thought that the physical applications of this theory are likely to lie in such areas as polymeric fluids and suspensions.

In the present note we shall consider the uniqueness of the solutions of the equations governing the isothermal motions of an incompressible anisotropic fluid with a director of constant (unit) magnitude. We assume that the fluid occupies a bounded region $D$ of threespace with a boundary $\partial D$ which is smooth enough to allow applications of the divergence theorem. If we introduce the director velocity $w_{i}$ by

$$
w_{i}=\dot{d}_{i},
$$

where a superposed dot denotes material time differentiation, then the fluid velocity $v_{i}$ and the director velocity $w_{1}$ satisfy the equations [2]

$$
\begin{gathered}
v_{i, i}=0, \\
\rho \dot{v}_{i}=\rho F_{i}-p_{, i}+\frac{1}{2} \alpha_{4} v_{i, j j}+\left\{\alpha_{1} d_{i} d_{j} d_{k} d_{p} v_{(k, p)}+\alpha_{2}\left(d_{j} w_{i}+v_{[k, i]} d_{j} d_{k}\right)\right. \\
\left.+\alpha_{3}\left(d_{i} w_{j}+v_{[k, j]} d_{i} d_{k}\right)+\alpha_{5} d_{j} d_{k} v_{(k, i)}+\alpha_{6} d_{i} d_{k} v_{(k, j)}\right\}_{, j} \\
\rho_{1} \dot{w}_{i}=\rho_{1} L_{i}-\gamma d_{i}-\left(\alpha_{3}-\alpha_{2}\right)\left[w_{i}+v_{[k, i]} d_{k}\right]-\left(\alpha_{6}-\alpha_{5}\right) d_{j} v_{(j, i)}
\end{gathered}
$$

in the space-time cylinder $D \times[0, T]$, where $T$ is a finite instant of time and

$$
v_{(i, j)}=\frac{1}{2}\left(v_{i, j}+v_{j, i}\right), \quad v_{[i, j]}=\frac{1}{2}\left(v_{i, j}-v_{j, i}\right) .
$$

Throughout this note we employ the usual convention of summing over repeated indices and a subscript $k$ following a comma indicates partial differentiation with respect to the space variable $x_{k}$. In equations (1.2) and (1.3), $\rho$ denotes the (constant) density, $\rho_{1}$ is a positive constant and $F_{i}$ and $L_{i}$ are the prescribed body forces. The unknown scalar functions $p$ and $\gamma$ are called the pressure and director tension, respectively, and they arise from the constraints of 
incompressibility and the director having a fixed magnitude. The coefficients $\alpha_{1}(i=1,2, \ldots, 6)$ are constants which, from thermodynamical considerations, are restricted to satisfy the conditions [2]

$$
\begin{aligned}
& \alpha_{4} \geqq 0, \quad 2 \alpha_{1}+3 \alpha_{4}+2 \alpha_{5}+2 \alpha_{6} \geqq 0, \quad 2 \alpha_{4}+\alpha_{5}+\alpha_{6} \geqq 0, \\
& \left(\alpha_{3}-\alpha_{2}\right) \geqq 0, \quad 4\left(\alpha_{3}-\alpha_{2}\right)\left(2 \alpha_{4}+\alpha_{5}+\alpha_{6}\right) \geqq\left(\alpha_{2}+\alpha_{3}+\alpha_{6}-\alpha_{5}\right)^{2} .
\end{aligned}
$$

For the purposes of this note it is convenient to introduce a new variable $\Omega_{i}$ termed the rotational velocity and defined by

$$
\Omega_{i}=\varepsilon_{i j k} d_{j} w_{k}, \quad w_{i}=\varepsilon_{i j k} \Omega_{j} d_{k}
$$

where $\varepsilon_{i j k}$ denotes the alternating tensor. Employing (1.5) $)_{2}$, we can rewrite (1.2) as

$$
\begin{aligned}
\rho \dot{v}_{i}= & \rho F_{i}-p, i+\frac{1}{2} \alpha_{4} v_{i, j j}+\left\{\alpha_{1} d_{i} d_{j} d_{k} d_{p} v_{(k, p)}+\alpha_{2}\left(\varepsilon_{i p k} \Omega_{p} d_{j} d_{k}+v_{[k, i]} d_{j} d_{k}\right)\right. \\
& \left.+\alpha_{3}\left(\varepsilon_{j p k} \Omega_{p} d_{i} d_{k}+v_{[k, j]} d_{i} d_{k}\right)+\alpha_{5} v_{(k, i)} d_{j} d_{k}+\alpha_{6} v_{(k, j)} d_{i} d_{k}\right\}_{, j}
\end{aligned}
$$

and moreover, by multiplying (1.3) by $\varepsilon_{k j i} d_{j}$ we obtain an equation that does not involve the director tension $\gamma$, namely,

$$
\rho_{1} \dot{\Omega}_{i}=\rho_{1} \varepsilon_{i j k} d_{j} L_{k}-\left(\alpha_{3}-\alpha_{2}\right)\left[\Omega_{i}+\varepsilon_{i j k} v_{[p, k]} d_{j} d_{p}\right]-\left(\alpha_{6}-\alpha_{5}\right) \varepsilon_{i j k} v_{(p, k)} d_{j} d_{p} .
$$

We shall restrict our attention to classical solutions which are assumed to exist in $[0, T)$ subject to prescribed initial conditions

$$
v_{i}(\mathbf{x}, 0)=f_{i}(\mathbf{x}), \quad d_{i}(\mathbf{x}, 0)=g_{i}(\mathbf{x}), \quad w_{i}(\mathbf{x}, 0)=h_{i}(\mathbf{x}) \quad \text { in } \quad D \times 0
$$

and, on $\partial D \times(0, T)$, prescribed boundary conditions of one of the following types:

$$
v_{i}(\mathbf{x}, t)=F_{i}(\mathbf{x}, t), \quad F_{i} n_{i}=0,
$$

or, if $F_{i} n_{i} \neq 0$,

$$
\begin{array}{lll}
v_{i}(\mathbf{x}, t)=F_{i}(\mathbf{x}, t), & d_{i}(\mathbf{x}, t)=G_{i}(\mathbf{x}, t), & w_{i}(\mathbf{x}, t)=H_{i}(\mathbf{x}, t), \\
v_{i}(\mathbf{x}, t)=F_{i}(\mathbf{x}, t), & d_{i}(\mathbf{x}, t)=G_{i}(\mathbf{x}, t), & \partial d_{i}(\mathbf{x}, t)=J_{i}(\mathbf{x}, t),
\end{array}
$$

where $n_{i}$ are the Cartesian components of the unit normal to $\partial D$ and $\partial$ is the normal gradient operator. In what follows we employ the familiar energy arguments to show that the fluid motion in $D$ is uniquely determined by the initial velocity, director and director velocity together with one of the sets of boundary conditions (1.9), (1.10) or (1.11).

2. Uniqueness. We shall say that a continuously differentiable pair $\left(v_{i}, d_{i}\right)$ is a solution to problem $\mathscr{A}$ if it satisfies the equations (1.1)-(1.3), the boundary conditions (1.9) and at $t=0$ reduces to the conditions $(1.8)_{1,2}$. Furthermore, if $\left(v_{i}, d_{i}\right)$ is a solution pair of problem $\mathscr{A}$ with an associated rotational velocity $\Omega_{i}$ and the pair $\left(v_{i}^{*}, d_{i}^{*}\right)$ a solution corresponding to the same body forces $F_{i}$ and $L_{i}$ with rotational velocity $\Omega_{i}^{*}$, then it is easily verified that the difference fields

$$
u_{i}=v_{i}-v_{i}^{*}, \quad D_{i}=d_{i}-d_{i}^{*}, \quad \mu_{i}=\Omega_{i}-\Omega_{i}^{*}
$$


satisfy the equations

and

$$
\begin{aligned}
\rho u_{i, t}= & -\rho v_{j} u_{i, j}-\rho v_{i, j}^{*} u_{j}-\left(p_{, i}-p_{, i}^{*}\right)+\frac{1}{2} \alpha_{4} u_{i, j j}+\alpha_{1} A_{i j, j} \\
& +\alpha_{2} B_{i j, j}+\alpha_{3} B_{j i, j}+\alpha_{5} C_{i j, j}+\alpha_{6} C_{j i, j}
\end{aligned}
$$

where

$$
\begin{aligned}
\rho_{1} \mu_{i, s}= & -\rho_{1} v_{j} \mu_{l, j}-\rho_{1} \Omega_{i, j}^{*} u_{j} \\
& -\left(\alpha_{3}-\alpha_{2}\right)\left[\mu_{i}-\varepsilon_{i j k} u_{[p, j]} d_{k} d_{p}-\varepsilon_{i j k} v_{[p, j]}^{*}\left(D_{k} d_{p}+D_{p} d_{k}^{*}\right)\right] \\
& +\left(\alpha_{6}-\alpha_{5}\right)\left[\varepsilon_{i j k} u_{(p, j)} d_{k} d_{p}+\varepsilon_{i j k} v_{(p, j)}^{*}\left(D_{k} d_{p}+D_{p} d_{k}^{*}\right)\right],
\end{aligned}
$$

$$
\begin{aligned}
& A_{i j}=u_{(k, p)} d_{i} d_{j} d_{k} d_{p}+v_{(k, p)}^{*}\left(D_{i} d_{j} d_{k} d_{p}+D_{j} d_{k} d_{p} d_{i}^{*}+D_{k} d_{p} d_{i}^{*} d_{j}^{*}+D_{p} d_{i}^{*} d_{j}^{*} d_{k}^{*}\right), \\
& B_{i j}=\varepsilon_{l p k} \mu_{p} d_{j} d_{k}+\varepsilon_{i p k} \Omega_{p}^{*}\left(D_{j} d_{k}+D_{k} d_{j}^{*}\right)+u_{[k, i]} d_{j} d_{k}+v_{[k, i]}^{*}\left(D_{j} d_{k}+D_{k} d_{j}^{*}\right), \\
& C_{i j}=u_{(k, i)} d_{j} d_{k}+v_{(k, i)}^{*}\left(D_{j} d_{k}+D_{k} d_{j}^{*}\right),
\end{aligned}
$$

and a comma followed by a $t$ indicates partial differentiation with respect to time, holding the spatial variable fixed. Moreover, from the definitions (1.5) and (2.1), we deduce

$$
D_{i} D_{i, i}=\varepsilon_{i j k} \mu_{j} D_{i} d_{k}-D_{i} D_{i, j} v_{j}-D_{i} u_{j} d_{i, j}^{*} \text {. }
$$

We are now in a position to establish the

THEOREM. If the coefficients $\alpha_{1}(i=1,2, \ldots, 6)$ satisfy (1.4) but with the strict inequality holding for $(1.4)_{1,2,3}$, then there is at most one solution of the initial value problem $\mathscr{A}$.

We first introduce the function $F(t)$ defined by

$$
F(t)=\int_{D_{i}}\left(\rho u_{i} u_{i}+\rho_{1} \mu_{i} \mu_{i}+D_{i} D_{i}\right) d x,
$$

where the symbol $D_{t}$ indicates that the integral is to be taken over the region $D$ at time $t$. Differentiation using the boundary conditions (1.9) yields

$$
F^{\prime}(t)=2 \int_{D_{\mathrm{t}}}\left(\rho u_{i} u_{i, t}+\rho_{1} \mu_{i} \mu_{i, t}+D_{i} D_{i, t}\right) d x
$$

Consequently, on using the equations (2.2)-(2.4) and integrating by parts, we obtain

$$
\begin{aligned}
F^{\prime}(t)= & -2 \int_{D_{t}}\left\{\rho u_{i} u_{j} v_{(i, j)}^{*}+\frac{1}{2} \alpha_{4} u_{i, j} u_{i, j}+\alpha_{1} u_{(i, j)} A_{i j}+\alpha_{2} u_{i, j} B_{i j}+\alpha_{3} u_{i, j} B_{j i}+\alpha_{5} u_{i, j} C_{i j}\right. \\
& +\alpha_{6} u_{i, j} C_{j i}+\rho_{1} \mu_{i} u_{j} \Omega_{i, j}^{*}+\left(\alpha_{3}-\alpha_{2}\right) \mu_{i}\left[\mu_{i}-\varepsilon_{i j k} u_{[p, j]} d_{k} d_{p}-\varepsilon_{i j k} v_{[p, j]}^{*}\left(D_{k} d_{p}+D_{p} d_{k}^{*}\right)\right] \\
& \left.-\left(\alpha_{6}-\alpha_{5}\right) \mu_{i} \varepsilon_{i j k}\left[u_{(p, j)} d_{k} d_{p}+v_{(p, j)}^{*}\left(D_{k} d_{p}+D_{p} d_{k}^{*}\right)\right]+D_{i} u_{j} d_{i, j}^{*}+\varepsilon_{i j k} \mu_{k} D_{i} d_{j}\right\} d x
\end{aligned}
$$


Rearranging the terms, we can write

where

$$
\begin{aligned}
F^{\prime}(t)=- & 2 \int_{D_{t}}\left\{\rho u_{i} u_{j} v_{(i, j)}^{*}+\rho_{1} \mu_{i} u_{j} \Omega_{i, j}^{*}+u_{j} D_{i} d_{i, j}^{*}+\varepsilon_{i j k} \mu_{k} D_{i} d_{j}\right. \\
& +\alpha_{1} u_{i, j} v_{(k, p)}^{*}\left(D_{i} d_{j} d_{k} d_{p}+D_{j} d_{k} d_{p} d_{i}^{*}+D_{k} d_{p} d_{i}^{*} d_{j}^{*}+D_{p} d_{i}^{*} d_{j}^{*} d_{k}^{*}\right) \\
& +\alpha_{2} u_{i, j}\left(\Omega_{p}^{*} \varepsilon_{i p k}+v_{[k, i]}^{*}\right)\left(D_{j} d_{k}+D_{k} d_{j}^{*}\right)+\alpha_{3} u_{i, j}\left(\Omega_{p}^{*} \varepsilon_{j p k}+v_{[k, i]}^{*}\right)\left(D_{i} d_{k}+D_{k} d_{i}^{*}\right) \\
& +\alpha_{5} u_{i, j} v_{(k, i)}^{*}\left(D_{j} d_{k}+D_{k} d_{j}^{*}\right)+\alpha_{6} u_{i, j} v_{(k, j)}^{*}\left(D_{i} d_{k}+D_{k} d_{i}^{*}\right) \\
& +\left(\alpha_{3}-\alpha_{2}\right) \mu_{p} v_{[i, j]}^{*} \varepsilon_{j p k}\left(D_{i} d_{k}+D_{k} d_{i}^{*}\right)+\left(\alpha_{6}-\alpha_{5}\right) \mu_{p} v_{(i, j)}^{*} \varepsilon_{j p k}\left(D_{i} d_{k}+D_{k} d_{i}^{*}\right) \\
& \left.+\left(\alpha_{3}-\alpha_{2}\right) \mu_{i} \mu_{j} d_{i} d_{j}\right\} d x-2 Q,
\end{aligned}
$$

and

$$
\begin{aligned}
Q= & \int_{D_{t}}\left\{\frac{1}{2} \alpha_{4} u_{i, j} u_{i, j}+\alpha_{1} u_{(i, j)} u_{(k, p)} d_{i} d_{j} d_{k} d_{p}+\left(\alpha_{5}+\alpha_{6}\right) u_{(i, j)} u_{(k, j)} d_{i} d_{k}\right. \\
& \left.+\left(\alpha_{2}+\alpha_{3}+\alpha_{6}-\alpha_{5}\right) u_{(i, j)} d_{i} N_{j}+\left(\alpha_{3}-\alpha_{2}\right) N_{i} N_{i}\right\} d x
\end{aligned}
$$

$$
N_{i}=\varepsilon_{i p k} \mu_{p} d_{k}+u_{[k, i]} d_{k} \text {. }
$$

We then proceed to obtain estimates for the terms in the braces on the right hand side of (2.5). We let $M$ be the generic notation for an upper bound and note that, although the number $M$ will differ for every estimate, nevertheless it is always possible to determine its size. We employ weighted arithmetic-geometric mean and Schwarz's inequalities to obtain

$$
F^{\prime}(t) \leqq-2 Q+\int_{D_{t}}\left\{M\left(u_{i} u_{i}+\mu_{i} \mu_{i}+D_{i} D_{i}\right)+\frac{2}{\lambda} u_{i, j} u_{i, j}\right\} d x
$$

Here $\lambda$ is an arbitrary positive constant which is to be prescribed, while $M$ depends on $\lambda$ and the bounds of $\Omega_{l}^{*}$ and the spatial derivatives of $v_{l}^{*}, d_{l}^{*}, \Omega_{l}^{*}$ during $[0, T]$.

Now, since $\alpha_{4}$ is assumed strictly positive, we can choose a value of $\lambda$ such that

$$
\alpha_{4}^{\prime}=\alpha_{4}-\frac{1}{\lambda}
$$

is nonnegative. Moreover, if the inequalities (1.4) hold with $\alpha_{4}^{\prime}$ replacing $\alpha_{4}$, then we can show that (see [2], (5.8))

$$
Q-\frac{1}{\lambda} \int_{D_{\mathbf{t}}} u_{i, j} u_{i, j} d x
$$

is nonnegative. Consequently, if we restrict ourselves to those materials for which the inequalities $(1.4)_{1,2,3}$ hold strictly, we can deduce that

$$
F^{\prime}(t) \leqq M F(t) .
$$

Thus, by integrating from $t=0$ to $t=T$ using the initial conditions, we conclude that

$$
F(T) \exp (-M T) \leqq 0 .
$$


It follows that $F(t)$ is zero for all $t \in[0, T]$ and thus $u_{i}=\mu_{i}=D_{i}=0$, so that the two flows are identical. Moreover, since $T$ is an arbitrary instant, we can conclude that the flows are identical as long as they exist.

We remark that, in the preceding proof, the boundary condition $(1.9)_{2}$ enabled us to show that the surface integrals

$$
\int_{\partial D_{t}} n_{i} v_{i} \mu_{k} \mu_{k} d x, \quad \int_{\partial D_{t}} n_{i} v_{i} D_{k} D_{k} d x
$$

are zero. Clearly these integrals will also vanish with boundary data (1.10). Moreover, given any surface, we can express the director velocity $w_{1}$ over the surface in terms of the surface gradient operator $\partial_{j}$ and the normal gradient operator $\partial$, thus:

$$
w_{i}=d_{i, t}+v_{j} \partial_{j} d_{i}+v_{j} n_{j} \partial d_{i}
$$

where $n_{i}$ is the unit normal to the surface, so that under data (1.11) the integrals (2.6) will again vanish. Consequently the corresponding uniqueness theorems for data (1.10) and (1.11) replacing (1.9) can be established.

\section{REFERENCES}

1. J. L. Ericksen, Anisotropic fluids, Arch. Rational Mech. Anal. 4 (1960), 231-237

2. F. M. Leslie, Some constitutive equations for anisotropic fluids, Quart. J. Mech. Appl. Math. 19 (1966), 357-370.

3. J. L. Ericksen, Continuum theory of liquid crystals, Appl. Mech. Rev. 20 (1967), 1029-1032.

HERIOT-WATT UNIVERSITY

EDINBURGH 